\title{
Intercellular Adhesion Molecule 1-Dependent Activation of Interleukin 8 Expression in Candida albicans-Infected Human Gingival Epithelial Cells
}

\author{
Hiroshi Egusa, ${ }^{1,2 *}$ Hiroki Nikawa, ${ }^{1}$ Seicho Makihira, ${ }^{1}$ Anahid Jewett, ${ }^{3}$ \\ Hirofumi Yatani, ${ }^{2}$ and Taizo Hamada ${ }^{1}$ \\ Department of Prosthetic Dentistry, Hiroshima University Graduate School of Biomedical Sciences, \\ Minami-ku, Hiroshima City, ${ }^{1}$ and Department of Fixed Prosthodontics, Osaka University \\ Graduate School of Dentistry, Suita City, Osaka, ${ }^{2}$ Japan, and The Jane and \\ Jerry Weintraub Center for Reconstructive Biotechnology, UCLA School \\ of Dentistry, Los Angeles, California ${ }^{3}$
}

Received 25 August 2004/Returned for modification 31 August 2004/Accepted 10 September 2004

\begin{abstract}
Increased induction of interleukin 8 (IL-8) and intercellular adhesion molecule 1 (ICAM-1) by oral epithelial cells may play a role in the host defense mechanism in oropharyngeal candidiasis; however, little is known about the expression feature of these molecules on human gingival epithelial cells (HGECs) during Candida albicans infection. In this report we present evidence that neutralization with antibody against ICAM-1 inhibited both the adherence of $C$. albicans to HGECs and the Candida-induced production of IL-8, suggesting a role for ICAM-1 in recognition and signaling in HGECs to express IL-8 upon infection with C. albicans.
\end{abstract}

Oral candidiasis is associated with significant morbidity (19) and may predispose severely immunocompromised patients to invasive disease (6). In recent years, the emergence of human immunodeficiency virus infection and the frequent use of immunosuppressant therapy have led to a sharp increase in the incidence of oral infections caused primarily by Candida albicans $(10,22,23)$.

In oral microbial infection, induction of a neutrophil chemoattractant, interleukin 8 (IL-8), and the adhesion molecule intercellular adhesion molecule 1 (ICAM-1) by oral epithelial cells may play an important role in recruiting and retaining neutrophils $(12,14)$. ICAM-1 is known not only as a cell-cell adhesion molecule (29), but also as a receptor on cells for microbial pathogens $(1,9,11,27,30)$. Furthermore, ICAM-1 has recently been shown to act as a signaling molecule to transmit outside-in signals in response to the binding of ligands, such as $\beta 2$-integrin (for a review, see reference 16). In addition, cross-linking of ICAM-1 on endothelial cells elevates mRNA and the IL-8 protein (25). On the other hand, several investigators have reported the existence of surface integrinlike proteins on $C$. albicans that are antigenically, structurally, and functionally related to the $\beta 2$-integrin (for a review, see reference 13). These observations prompted us to explore the possibility that ICAM-1 plays a role in mediating $C$. albicans and human gingival epithelial cell (HGEC) interaction to transmit outside-in signals to induce the expression of IL-8.

We used the oral isolate C. albicans ATCC MYA-273, grown in yeast nitrogen base broth (Difco, Detroit, Mich.) containing $250 \mathrm{mM}$ glucose at $37^{\circ} \mathrm{C}$ for $18 \mathrm{~h}$ with shaking at $100 \mathrm{rpm}$. The cells were separated from the medium by centrifugation at

\footnotetext{
* Corresponding author. Mailing address: Department of Fixed Prosthodontics, Osaka University Graduate School of Dentistry, 1-8 Yamadaoka, Suita City, Osaka 565-0871, Japan, Phone: 81-6-68792946. Fax: 81-6-6879-2947. E-mail: egu@dent.osaka-u.ac.jp.
}

$3,000 \times g$ for $5 \mathrm{~min}$, washed three times with phosphatebuffered saline (PBS), and finally resuspended in fresh keratinocyte basal medium (Clonetics, San Diego, Calif.) for each experiment (20). Primary HGEC cultures were established from discarded healthy gingival tissues at surgery with the donors' informed consent. Briefly, the tissues were treated overnight with $0.025 \%$ trypsin and $0.02 \%$ EDTA at $4^{\circ} \mathrm{C}$. After trypsin neutralization and washing, the epithelial layer was separated from the underlying connective tissue and HGECs were collected by scraping the epithelial layer in keratinocyte growth medium (Clonetics) (20). The cell suspension was seeded in plastic tissue culture plates coated with type I collagen and maintained at $37^{\circ} \mathrm{C}$ in an atmosphere of $5 \% \mathrm{CO}_{2}$. All experiments were performed with second- and third-passage HGECs.

We first examined by real-time reverse transcription-PCR (RT-PCR) analysis whether HGECs upregulate IL-8 and ICAM-1 expression in response to $C$. albicans infection. HGECs were grown to confluence in 48-well culture plates. After we changed the medium to fresh keratinocyte growth medium lacking amphotericin $\mathrm{B}$, cells were challenged with $200 \mu \mathrm{l}$ of $10^{5}$ cells of $C$. albicans per $\mathrm{ml}$ of suspension and maintained for $8 \mathrm{~h}$. Total RNA from HGECs was prepared by using an RNeasy Mini kit (QIAGEN, Hilden, Germany), followed by DNase treatment (Ambion, Austin, Tex.). cDNA was synthesized from $1 \mu \mathrm{g}$ of total RNA with Omniscript reverse transcriptase (QIAGEN). PCR amplification and detection of target genes and the internal-control glyceraldehyde-3-phosphate dehydrogenase (GAPDH) gene were performed by using the ABI PRISM 7700 sequence detection system (PerkinElmer Applied Biosystems, Foster City, Calif.). The primers and probes for IL-8 and GAPDH used in this study are commercially available and were obtained from PE Applied Biosystems. Those for ICAM-1 were designed with the Primer Express software program (PE Applied Biosystems) (5'-ACC 

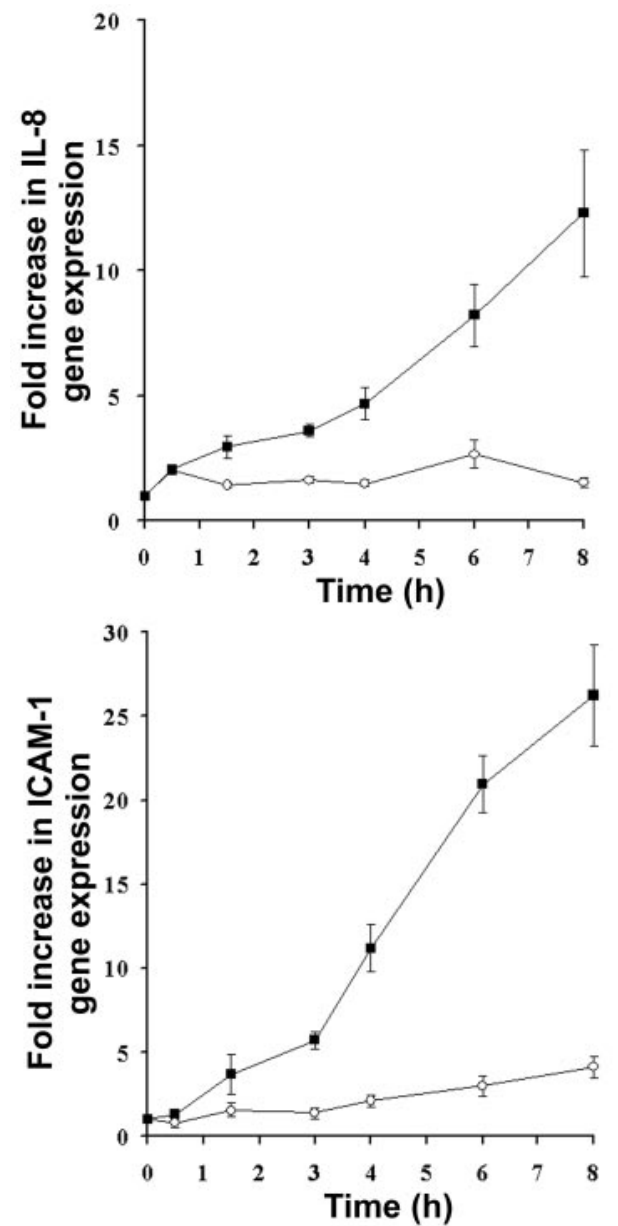

FIG. 1. Changes in IL-8 and ICAM-1 transcript levels in response to $C$. albicans infection in HGECs. Total RNA was isolated from $C$. albicans-infected HGECs $(\boldsymbol{\square})$ or uninfected HGECs $(\bigcirc)$ at the indicated time points, and levels of transcripts of IL-8 and ICAM-1 were determined by real-time RT-PCR analysis. Results represent the means \pm standard errors of the means ( $n$-fold increases) of results from three separate experiments.

TCA GCC TCG CTA TGG CT-3', 5'-ACA GGA GGT GCT GCA TGT CA-3', and 5'-FAM-GCT CTG TTC CCA GGA CCT GGC AAT G-TAMRA-3' [where FAM and TAMRA are 5-carboxyfluorescein and 6-carboxytetramethylrhodamine, respectively]). Final quantitation was derived using the comparative threshold cycle method (7).

When HGECs were incubated with $C$. albicans, expression levels of both IL- 8 and ICAM-1 continually increased as much as 12 - and 26-fold, respectively, at $8 \mathrm{~h}$. In contrast, no or slight changes in the expression of these genes were observed over the course of $8 \mathrm{~h}$ in the control cultures (Fig. 1). Previous reports have shown that $C$. albicans triggers IL-8 synthesis by oral epithelial cells $(5,26)$ and stimulates ICAM-1 expression by endothelial cells $(8,21)$. We found that $C$. albicans stimulated the accumulation of mRNAs for IL-8 and ICAM-1 in HGECs, and mRNA accumulation continued to increase until at least $8 \mathrm{~h}$ after infection. IL- 8 activates neutrophils and monocytes to kill fungal targets (4). The loss of ICAM-1 in deficient mice significantly impaired the host defense against
C. albicans by impairing neutrophil migration and/or phagocyte activation (2). Presumably, the local expression of both IL- 8 and ICAM-1 by HGECs plays a critical role in the host defense against $C$. albicans.

We found in preliminary experiments using scanning electron microscopy that HGECs exhibited lamellar extensions that were in intimate contact with cell walls of $C$. albicans germ tubes at $5 \mathrm{~h}$ postinfection (Fig. 2A). To examine whether the expression of ICAM-1 molecules is associated with the contact site of HGECs with $C$. albicans, we performed immunocytochemistry experiments.

HGECs were grown on 25-mm-diameter circular glass coverslips coated with type I collagen. When cells reached $50 \%$ confluence, cells were challenged with $100 \mu \mathrm{l}$ of $10^{4}$ cells of $C$. albicans per $\mathrm{ml}$ of suspension and maintained for $5 \mathrm{~h}$. Subsequently, samples were washed with PBS and fixed in $2 \%$ paraformaldehyde for $10 \mathrm{~min}$. Cells were incubated for $20 \mathrm{~min}$ in blocking solution containing $1 \%$ bovine serum albumin and $0.1 \%$ Triton X-100 and then washed and incubated for $1 \mathrm{~h}$ at $37^{\circ} \mathrm{C}$ with $5 \mu \mathrm{g}$ of mouse anti-human ICAM-1 monoclonal antibody (MAb) (84H10; Chemicon, Temecula, Calif.) per ml in PBS containing $1 \%$ bovine serum albumin. To assess nonspecific staining, a mouse immunoglobulin $\mathrm{G}(\mathrm{IgG})$ whole molecule (Chemicon) was used at the same concentration as the primary antibody. Cells were then washed and incubated for 30 $\min$ at $37^{\circ} \mathrm{C}$ with fluorescein isothiocyanate-conjugated rabbit anti-mouse IgG (1:100; Sigma), employed to detect ICAM-1. Washed cells were viewed with laser scanning microscopes (model LSM410; Carl Zeiss, Inc., Germany).

Although staining intensities were not significantly different between C. albicans-infected and uninfected HGECs (Fig. 2C and $\mathrm{G}$ ), we observed that ICAM-1 was concentrated in the region immediately adjacent to the $C$. albicans cell, so that the germ tubes were outlined by ICAM-1 (Fig. 2G). Control IgG did not stain the $C$. albicans-infected HGECs (Fig. 2E). Similar findings have been reported for $C$. albicans-infected endothelial cells showing ICAM-1 that has accumulated around a candidal germ tube (8). Based on these observations, ICAM-1 appears to play a role in the interaction with $C$. albicans at the contact site. Therefore, we further examined the relationship between $C$. albicans and ICAM-1 on HGECs by MAb neutralization experiments.

Antiadhesion antibody assay was performed to determine whether $C$. albicans binds to HGECs via ICAM-1. When HGECs plated on collagen-coated 96-well culture plates were grown to confluence, cells were preincubated for $30 \mathrm{~min}$ with $20 \mu \mathrm{g}$ of antibody solution (ICAM-1 or control IgG) per ml. $C$. albicans was then added at $5 \times 10^{4}$ cells per well, and the plates were incubated for $30 \mathrm{~min}$. Subsequently, the cell culture supernatants were removed and washed three times with PBS for $30 \mathrm{~s}$ with gentle agitation at $100 \mathrm{rpm}$ to dislodge the loosely adherent yeast cells (20). The monolayer with the attached yeast cells was fixed in $10 \%$ buffered formalin phosphate and Gram stained (24). Finally, adherent yeast cells were counted under a phase-contrast microscope at a $\times 200$ magnification, with the visual field being set in the center of each well.

The antiadhesion antibody assay demonstrated a significant inhibition effect of anti-ICAM-1 MAb on the adhesion of $C$. albicans to HGECs, reducing overall adhesion to about $67 \%$, whereas control $\mathrm{IgG}$ did not significantly affect the adhesion 

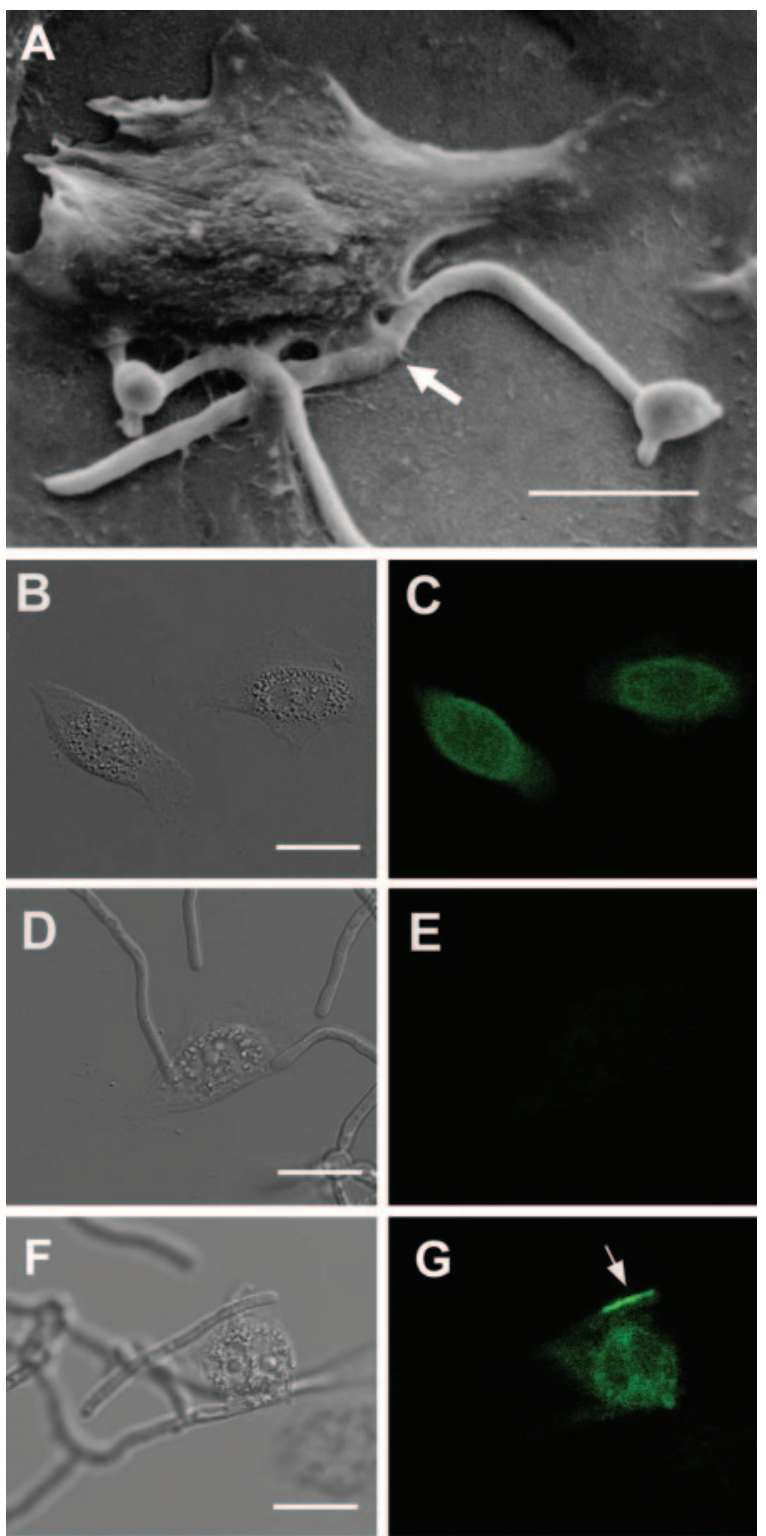

FIG. 2. Microscopic observation of the contact site of HGECs with C. albicans. HGECs were challenged with $C$. albicans and incubated for $5 \mathrm{~h}$. (A) Scanning electron micrograph. Note the lamellar extensions of the HGECs that are in intimate contact with cell walls of $C$. albicans germ tubes (arrow). Bar, $10 \mu \mathrm{m}$. (B, D, and F) Differential interference contrast micrographs. (C, E, and $\mathrm{G}$ ) Immunocytochemical analysis showing the same areas as in panels B, D, and F, respectively. Bars, $10 \mu \mathrm{m}$. (C and $\mathrm{G}$ ) The surface expression of ICAM-1 in noninfected (C) or infected (G) HGECs was detected by immunocytochemistry. Note the accumulation of ICAM-1 around the candidal germ tube in panel G (arrow). (E) Mouse IgG (whole molecule) was used as a negative control.

(Fig. 3). Addition of anti-ICAM-1 MAb at a concentration of $20 \mu \mathrm{g} / \mathrm{ml}$ in the absence of C. albicans did not effect the expression of IL-8 mRNA by HGECs (Fig. 4). Although antiICAM-1 antibody is known to inhibit T-cell adhesion and immune function (31), it seems that the anti-ICAM-1 MAb used in this study did not modify the activation of HGECs but inhibited the direct binding of $C$. albicans with HGECs. Our



FIG. 3. Effect of anti-ICAM-1 MAb on the adherence of C. albicans to HGECs. Results shown are mean percentages of adherent yeast cells compared to the percentage for the nontreated group \pm standard errors of the means. The experiment was carried out in triplicate on three separate occasions (nine replicates). *, significant difference compared with the value for the nontreated control group $(P<0.01$, analysis of variance [Dunnett's multiple-comparison test]).

result supported a previous report that ICAM-1 plays a role in the adherence of $C$. albicans to pulmonary vascular endothelial cells (34). In both studies, however, inhibition of C. albicans adhesion to host cells by anti-ICAM-1 antibody was not complete. Some investigators have shown that integrin-like proteins in $C$. albicans bind to RGD sequences expressed in epithelial cells (13). Therefore, it is thought that there are $C$. albicans binding sites other than ICAM-1 on the surfaces of these cells. Nonetheless, our results suggest that ICAM-1 plays, in part, a role in the attachment of $C$. albicans to HGECs. These observations may explain the preference for candidal adherence to cancer epithelial cells (28) that highly express ICAM-1 (15). Another study reported that fimbrial binding of Porphyromonas gingivalis to HGECs occurs through ICAM-1 (33). Other microbial pathogens, such as human rhinovirus (30), coxsackievirus group A (27), and Plasmodium falciparum (9), are also able to bind to this molecule. ICAM-1 may partly be involved in the adhesion of a variety of microorganisms to host cells, which likely leads to colonization and/or invasion of the organisms in host tissues.

Since our results suggest the binding of $C$. albicans to HGECs via ICAM-1 and the strong expression of ICAM- 1 and IL-8, it is of interest to examine the role of ICAM-1 in signal transduction related to the $C$. albicans-induced expression of IL-8 mRNA in HGECs. To investigate this possibility, HGECs were precultured for $30 \mathrm{~min}$ with 5 to $20 \mu \mathrm{g}$ of anti-ICAM-1 $\mathrm{MAb}$ or control IgG per $\mathrm{ml}$ and then challenged with $200 \mu \mathrm{l}$ of $10^{5}$ cells of $C$. albicans per $\mathrm{ml}$ of suspension, followed by $8 \mathrm{~h}$ of incubation. Total RNA was extracted from each sample, and mRNA levels for IL-8 were determined by real-time RT-PCR analysis. When HGECs were cultured in the presence of more than $10 \mu \mathrm{g}$ of anti-ICAM-1 MAb per ml, increased expression of $C$. albicans-induced IL-8 mRNA was decreased dose dependently. On the other hand, the expression of $C$. albicans-in- 


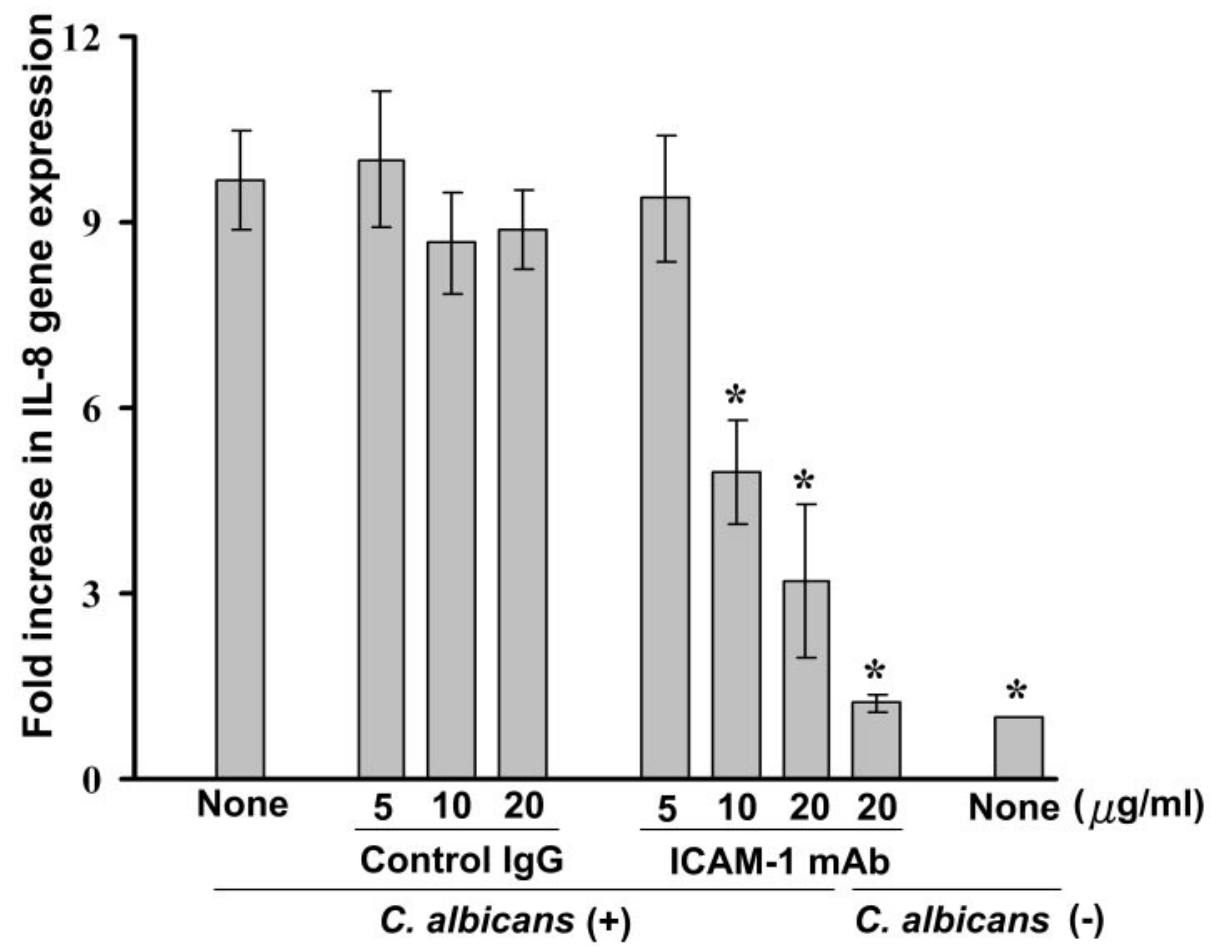

FIG. 4. Neutralization with anti-ICAM-1 MAb inhibits the expression of IL-8 mRNA in C. albicans-infected HGECs. HGECs were preincubated with 5,10, or $20 \mu \mathrm{g}$ of control IgG or anti-ICAM-1 MAb per ml for $30 \mathrm{~min}$, followed by culture with C. albicans or medium alone for $8 \mathrm{~h}$. Total RNA was isolated from each sample, and levels of IL-8 transcript were determined by real-time RT-PCR analysis. Values given are means \pm standard errors of the means ( $n$-fold increases) in the IL- 8 gene expression level compared to that in uninfected HGECs for three separate experiments. *, significant difference compared with the value for the group of HGECs infected with $C$. albicans without MAb treatment $(P<0.01$, analysis of variance [Dunnett's multiple-comparison test]).

duced IL-8 did not significantly change in the presence of control IgG (Fig. 4).

These results, taken together, suggest that the binding of HGECs to $C$. albicans through the ICAM-1 molecule induces the expression of IL- 8 mRNA, implying that ICAM-1 functions as a signaling molecule after binding with $C$. albicans to transmit outside-in signals in HGECs. It has been reported that the stimulation of ICAM-1 induces the production of cytokines, such as IL-8 in endothelial cells (25) and IL-1 $\beta$ in synovial cells (18). A signal may be delivered by ICAM-1 to the cells in response to the binding of ligands, such as $\beta 2$ integrin (16). $C$. albicans expresses $\beta 2$ integrin-like proteins involved in adhesion and morphogenesis on the cell surface (13). Therefore, it is possible that the $\beta 2$ integrin-like proteins on $C$. albicans bind through ICAM-1 expressed on HGECs, resulting in the activation of a signaling cascade for the induction of IL-8 gene transcription. However, since the mechanisms involved in the ICAM-1-mediated activation of IL- 8 mRNA expression are not clear, further studies are warranted to identify the cascade. Recently, a critical role played by Toll-like receptors 2 and 4 in C. albicans-induced cell signaling pathways has been suggested $(3,17,32)$. In addition to these molecules, we now demonstrate that ICAM-1 can play a vital role in recognition and signaling in HGECs upon infection with C. albicans.

In conclusion, the present study found that ICAM-1 can play a role in the binding of $C$. albicans and the subsequent induction of the expression of the IL- 8 gene in HGECs, which likely contributes to the effective recruitment and activation of neutrophils locally as well as the prevention of disseminated infection. Our study offers a deeper insight into the host cellCandida interaction, which may lead to the development of effective therapeutic strategies.

\section{REFERENCES}

1. Berendt, A. R., A. McDowall, A. G. Craig, P. A. Bates, M. J. Sternberg, K. Marsh, C. I. Newbold, and N. Hogg. 1992. The binding site on ICAM-1 for Plasmodium falciparum-infected erythrocytes overlaps, but is distinct from, the LFA-1-binding site. Cell 68:71-81.

2. Davis, S. L., E. P. Hawkins, E. O. Mason, Jr., C. W. Smith, and S. L. Kaplan. 1996. Host defenses against disseminated candidiasis are impaired in intercellular adhesion molecule 1-deficient mice. J. Infect. Dis. 174:435-439.

3. Deva, R., P. Shankaranarayanan, R. Ciccoli, and S. Nigam. 2003. Candida albicans induces selectively transcriptional activation of cyclooxygenase- 2 in HeLa cells: pivotal roles of Toll-like receptors, p38 mitogen-activated protein kinase, and NF-kappa B. J. Immunol. 171:3047-3055.

4. Djeu, J. Y., K. Matsushima, J. J. Oppenheim, K. Shiotsuki, and D. K. Blanchard. 1990. Functional activation of human neutrophils by recombinant monocyte-derived neutrophil chemotactic factor/IL-8. J. Immunol. 144: 2205-2210.

5. Dongari-Bagtzoglou, A., and H. Kashleva. 2003. Candida albicans triggers interleukin-8 secretion by oral epithelial cells. Microb. Pathog. 34:169-177.

6. Edwards, J. E., Jr., R. I. Lehrer, E. R. Stiehm, T. J. Fischer, and L. S. Young. 1978. Severe candidal infections: clinical perspective, immune defense mechanisms, and current concepts of therapy. Ann. Intern. Med. 89:91-106.

7. Fehniger, T. A., M. H. Shah, M. J. Turner, J. B. VanDeusen, S. P. Whitman, M. A. Cooper, K. Suzuki, M. Wechser, F. Goodsaid, and M. A. Caligiuri. 1999. Differential cytokine and chemokine gene expression by human NK cells following activation with IL-18 or IL-15 in combination with IL-12: implications for the innate immune response. J. Immunol. 162:4511-4520.

8. Filler, S. G., A. S. Pfunder, B. J. Spellberg, J. P. Spellberg, and J. E. Edwards, Jr. 1996. Candida albicans stimulates cytokine production and 
leukocyte adhesion molecule expression by endothelial cells. Infect. Immun 64:2609-2617.

9. Gray, C., C. McCormick, G. Turner, and A. Craig. 2003. ICAM-1 can play a major role in mediating $\mathrm{P}$. falciparum adhesion to endothelium under flow. Mol. Biochem. Parasitol. 128:187-193.

10. Greenwood, I., J. M. Zakrzewska, and P. G. Robinson. 2002. Changes in the prevalence of HIV-associated mucosal disease at a dedicated clinic over 7 years. Oral Dis. 8:90-94.

11. Greve, J. M., G. Davis, A. M. Meyer, C. P. Forte, S. C. Yost, C. W. Marlor, M. E. Kamarck, and A. McClelland. 1989. The major human rhinovirus receptor is ICAM-1. Cell 56:839-847.

12. Han, D. C., G. T. Huang, L. M. Lin, N. A. Warner, J. S. Gim, and A. Jewett. 2003. Expression of MHC class II, CD70, CD80, CD86 and pro-inflammatory cytokines is differentially regulated in oral epithelial cells following bacterial challenge. Oral Microbiol. Immunol. 18:350-358.

13. Hostetter, M. K. 1999. Integrin-like proteins in Candida spp. and other microorganisms. Fungal Genet. Biol. 28:135-145.

14. Huang, G. T., S. K. Haake, J. W. Kim, and N. H. Park. 1998. Differentia expression of interleukin-8 and intercellular adhesion molecule-1 by human gingival epithelial cells in response to Actinobacillus actinomycetemcomitans or Porphyromonas gingivalis infection. Oral Microbiol. Immunol. 13: 301-309.

15. Huang, G. T., X. Zhang, and N. H. Park. 2000. Increased ICAM-1 expression in transformed human oral epithelial cells: molecular mechanism and functional role in peripheral blood mononuclear cell adhesion and lymphokineactivated-killer cell cytotoxicity. Int. J. Oncol. 17:479-486.

16. Hubbard, A. K., and R. Rothlein. 2000. Intercellular adhesion molecule-1 (ICAM-1) expression and cell signaling cascades. Free Radic. Biol. Med. 28:1379-1386.

17. Jouault, T., S. Ibata-Ombetta, O. Takeuchi, P. A. Trinel, P. Sacchetti, P. Lefebvre, S. Akira, and D. Poulain. 2003. Candida albicans phospholipomannan is sensed through toll-like receptors. J. Infect. Dis. 188:165-172.

18. Koyama, Y., Y. Tanaka, K. Saito, M. Abe, K. Nakatsuka, I. Morimoto, P. F Auron, and S. Eto. 1996. Cross-linking of intercellular adhesion molecule 1 (CD54) induces AP-1 activation and IL-1beta transcription. J. Immunol. 157:5097-5103.

19. Lynch, D. P. 1994. Oral candidiasis. History, classification, and clinical presentation. Oral Surg. Oral Med. Oral Pathol. 78:189-193.

20. Nikawa, H., H. Egusa, S. Makihira, M. Nishimura, K. Ishida, M. Furukawa, and T. Hamada. 2003. A novel technique to evaluate the adhesion of Candida species to gingival epithelial cells. Mycoses 46:384-389.

21. Orozco, A. S., X. Zhou, and S. G. Filler. 2000. Mechanisms of the proinflammatory response of endothelial cells to Candida albicans infection. Infect. Immun. 68:1134-1141.
22. Phelan, J. A., M. D. Begg, I. B. Lamster, J. Gorman, D. Mitchell-Lewis, R. D. Bucklan, and W. M. el-Sadr. 1997. Oral candidiasis in HIV infection: predictive value and comparison of findings in injecting drug users and homosexual men. J. Oral Pathol. Med. 26:237-243.

23. Robinson, P. G. 1997. The oral manifestations of HIV infection. Int. J. STD AIDS 8:668-674.

24. Samaranayake, L. P., and T. W. MacFarlane. 1981. The adhesion of the yeast Candida albicans to epithelial cells of human origin in vitro. Arch. Oral Biol. 26:815-820.

25. Sano, H., N. Nakagawa, R. Chiba, K. Kurasawa, Y. Saito, and I. Iwamoto. 1998. Cross-linking of intercellular adhesion molecule-1 induces interleukin- 8 and RANTES production through the activation of MAP kinases in human vascular endothelial cells. Biochem. Biophys. Res. Commun. 250: 694-698.

26. Schaller, M., R. Mailhammer, G. Grassl, C. A. Sander, B. Hube, and H. C. Korting. 2002. Infection of human oral epithelia with Candida species induces cytokine expression correlated to the degree of virulence. J. Investig. Dermatol. 118:652-657.

27. Shafren, D. R., D. J. Dorahy, R. A. Ingham, G. F. Burns, and R. D. Barry. 1997. Coxsackievirus A21 binds to decay-accelerating factor but requires intercellular adhesion molecule 1 for cell entry. J. Virol. 71:4736-4743.

28. Skoutelis, A. T., P. E. Lianou, E. Votta, H. P. Bassaris, and J. T. Papavassiliou. 1994. Adherence of Candida albicans to epithelial cells from normal and cancerous urinary bladders. Int. Urol. Nephrol. 26:519-522.

29. Springer, T. A. 1990. Adhesion receptors of the immune system. Nature 346:425-434.

30. Staunton, D. E., V. J. Merluzzi, R. Rothlein, R. Barton, S. D. Marlin, and T. A. Springer. 1989. A cell adhesion molecule, ICAM-1, is the major surface receptor for rhinoviruses. Cell 56:849-853.

31. Tibbetts, S. A., C. Chirathaworn, M. Nakashima, D. S. Jois, T. J. Siahaan, M. A. Chan, and S. H. Benedict. 1999. Peptides derived from ICAM-1 and LFA-1 modulate $\mathrm{T}$ cell adhesion and immune function in a mixed lymphocyte culture. Transplantation 68:685-692.

32. Villamon, E., D. Gozalbo, P. Roig, J. E. O'Connor, D. Fradelizi, and M. L. Gil. 2004. Toll-like receptor-2 is essential in murine defenses against Candida albicans infections. Microbes Infect. 6:1-7.

33. Wang, P. L., M. Shinohara, N. Murakawa, M. Endo, S. Sakata, M. Okamura, and K. Ohura. 1999. Effect of cysteine protease of Porphyromonas gingivalis on adhesion molecules in gingival epithelial cells. Jpn. J. Pharmacol. 80:75-79.

34. Yokomura, I., Y. Iwasaki, K. Nagata, M. Nakanishi, A. Natsuhara, H. Harada, Y. Kubota, M. Ueda, T. Inaba, and M. Nakagawa. 2001. Role of intercellular adhesion molecule 1 in acute lung injury induced by candidemia. Exp. Lung Res. 27:417-431.

Editor: T. R. Kozel 